\title{
Identification of Novel QTL Conferring Sheath Blight Resistance in Two Weedy Rice Mapping Populations
}

\author{
David M. Goad', Yulin Jia ${ }^{2}$, Andrew Gibbons ${ }^{3,4}$, Yan Liư ${ }^{5}$, David Gealy², Ana L. Caicedo ${ }^{6}$ and Kenneth M. Olsen ${ }^{1}$
}

\begin{abstract}
Background: Rice sheath blight (ShB) disease, caused by the pathogenic fungus Rhizoctonia solani, causes significant yield losses globally. US weedy rice populations, which are de-domesticated forms of indica and aus cultivated rice, appear to be more resistant to ShB than local japonica cultivated rice. We mapped quantitative trait loci (QTL) associated with $\mathrm{ShB}$ resistance using two $\mathrm{F}_{8}$ recombinant inbred line populations generated from crosses of an indica crop variety, Dee-Geo-Woo-Gen (DGWG), with individuals representing the two major US weed biotypes, straw hull $(\mathrm{SH})$ and black hull awned (BHA).

Results: We identified nine ShB resistance QTL across both mapping populations. Five were attributable to alleles that affect plant height $(\mathrm{PH})$ and heading date $(\mathrm{HD})$, two growth traits that are known to be highly correlated with ShB resistance. By utilizing an approach that treated growth traits as covariates in the mapping model, we were able to infer that the remaining four QTL are involved in ShB resistance. Two of these, qShB1-2 and qShB4, are different from previously identified ShB QTL and represent new candidates for further study.

Conclusion: Our findings suggest that ShB resistance can be improved through favorable plant growth traits and the combined effects of small to moderate-effect resistance QTL. Additionally, we show that including PH and HD as covariates in QTL mapping models is a powerful way to identify new ShB resistance QTL.
\end{abstract}

Keywords: Quantitative trait loci, Sheath blight disease, Rice, Genotyping-by-sequencing, Weeds

\section{Background}

Rice sheath blight (ShB) disease, caused by the soil borne fungus Rhizoctonia solani (teleomorph: Thanatephorus cucumeris), is one of the most devastating pathogens of rice worldwide (Savary et al. 2006). In the United States, it can cause up to $50 \%$ yield loss in infected fields (Rush and Lee 1983). The pathogen infects the plants at the waterline and spreads upwards, creating lesions on the leaf blades and sheaths. Infection to any detectable degree reduces yield, but the fungus is particularly destructive if it reaches the panicle and infects the grains. Because of its worldwide

\footnotetext{
* Correspondence: Yulin.Jia@USDA.GOV

2United States Department of Agriculture-Agricultural Research Service, Dale Bumpers National Rice Research Center, 2890 HWY 130 E, Stuttgart, AR

72160, USA

Full list of author information is available at the end of the article
}

impact, there have been many attempts to identify genes in rice that confer increased ShB resistance. However, to date, few major $\mathrm{ShB}$ resistance genes have been identified from either cultivated rice or wild rice relatives (Molla et al. 2019). Over 25 quantitative trait locus (QTL) mapping studies have been performed using crosses from a diverse set of parents, including improved cultivars (Liu et al. 2009; Li et al. 1995; Zou et al. 2000), landraces (Xu et al. 2011; Taguchi-Shiobara et al. 2013), and the wild rice species Oryza nivara (Eizenga et al. 2013) and O. meridonalis (Eizenga et al. 2015). These studies have produced a wealth of putative small-effect QTL; however, only a few of these loci, such as $q S B-9^{T Q}$ (Zuo et al. 2014) and $q S B$ $11^{L E}$ (Zuo et al. 2013) have been fine mapped and used for breeding. There is an urgent need to manage ShB by 
identifying novel sources of disease resistance and implementing them in management or breeding programs.

Mapping traits in new and diverse populations is one of the best ways to identify additional resistance QTL. So far, weedy rice accessions have not been used in developing ShB-resistant mapping populations despite their potential to harbor unique resistance alleles (Liu et al. 2015). Weedy rice is often the result of de-domestication of cultivated forms of rice (Oryza sativa) that occurs in rice production areas worldwide and aggressively outcompetes its domesticated relative (Wedger and Olsen 2018). In the US, two major biotypes of weedy rice are most common; they both have a red pericarp, but are largely distinguishable by grain hull characteristics and are referred to as straw hull (SH) and black hull awned (BHA) types (Londo and Schaal 2007). The SH and BHA weed biotypes are genetically distinct (Londo and Schaal 2007) and have been shown to have evolved independently by de-domestication from cultivated Asian rice varieties (SH from indica rice, BHA from aus rice) (Reagon et al. 2010; Li et al. 2017). Several factors suggest that these weeds could be promising sources of $\mathrm{ShB}$ resistance genes such as: 1) The $\mathrm{SH}$ and BHA strains are among the most predominant weeds in southern US rice fields where sheath-blight is the most destructive pathogen (Wrather and Sweets 2009), which suggests that they may possess a mechanism of disease resistance that confers a competitive advantage. 2) Weed $\times$ crop mapping populations for both biotypes have already been used to identify resistance QTL for another fungal disease (rice blast) (Liu et al. 2015). 3) Finally, because the two weed biotypes evolved independently and have historically shown limited hybridization with US cultivated rice (Reagon et al. 2010), any resistance alleles that they carry are likely to be unique to the weeds and unlikely to have been previously identified.

A complication in ShB-resistance genetic mapping studies is that the level of infection shown by a plant is correlated not only with resistance directly interacting with the pathogen but also with plant growth traits, particularly plant height (PH) and heading date (HD) (Li et al. 1995; Zou et al. 2000; Channamallikarjuna et al. 2010). ShB resistance has been typically scored on a $0-9$ or 1-9 scale by measuring the proportion of the stem above the waterline with signs of infection with 1 being very resistant and 9 being very susceptible. With this scoring system, greater $\mathrm{PH}$ is directly correlated with a higher resistance score ( $\mathrm{Li}$ et al. 1995). In the case of HD, the cause of the correlation is less well understood. It has been suggested that laterheading varieties are more resistant because they grow later in the season, when conditions are drier and less favorable for pathogen spread (Wasano and Hirota 1986), but this hypothesis has not been formally tested. While these growth traits may be of interest for selecting varieties in areas with severe ShB, they confound attempts to genetically map QTL for ShB resistance. Indeed, in most previous mapping studies of $\mathrm{ShB}$ resistance, the largest effect QTL have been directly attributable to loci for either $\mathrm{PH}$ or HD (as reviewed by Zeng et al. 2015). The confounding effects of growth traits with resistance could be particularly problematic for weed $\times$ crop mapping populations, as weedy rice differs dramatically from cultivated rice in both traits. However, it is possible to factor out these confounding effects if QTL mapping models that explicitly incorporate $\mathrm{PH}$ and $\mathrm{HD}$ measurements into the analysis as covariates are employed. Despite the potential utility to detect QTL associated with resistance, covariate modeling has been underutilized in the ShB QTL mapping literature, with (Nelson et al. 2012) as the sole example of this strategy.

In this study, we map QTL associated with ShB resistance using two weed $\times$ crop $\mathrm{F}_{8}$ mapping populations (derived from $\mathrm{SH} \times$ crop and $\mathrm{BHA} \times$ crop crosses) that were assessed in field conditions over two growing seasons. By using $\mathrm{PH}$ and $\mathrm{HD}$ measurements as covariates, we were able to remove the effects of QTL associated with these confounding growth traits and identify novel ShB resistance QTL and interactions that would otherwise have been undetected. Our SNP linkage maps for these populations yielded greater genetic resolution than previous mapping studies and have allowed us to identify potential candidate genes within our QTL confidence intervals. The relationship between $\mathrm{PH}, \mathrm{HD}$, and $\mathrm{ShB}$ confirmed in this study also has implications for optimal cultivar choice in regions of high $\mathrm{ShB}$ incidence and management practices in fields with recurring infestations.

\section{Methods}

\section{Inoculum Preparation}

To produce the required amounts of $R$. solani, a slow growing field isolate (RR0134) was chosen. The isolate was grown on a potato dextrose agar (PDA) by introducing shredded mycelium-infiltrated filter paper to the culture plate. The plate was incubated at $30^{\circ} \mathrm{C}$ until the appearance of black-bodied sclerotia. This product was used as the initial inoculants. To grow large amounts of $R$. solani, a mixture of corn, rye, and water in the proportion of 2.48 $\mathrm{kg}: 1.27 \mathrm{~kg}: 3.5$ to $3.75 \mathrm{l}(\mathrm{L})$, respectively was mixed and allowed to soak for $30 \mathrm{~min}$. The mixture was then autoclaved for $1 \mathrm{~h}$ at $121^{\circ} \mathrm{C} / 1.0 \mathrm{~kg} / \mathrm{cm}^{2}$. After the media were allowed to cool overnight, they were mixed and doublebagged. The double-bagged media were loosely sealed and autoclaved an additional two cycles $\left(1 \mathrm{~h} / 121^{\circ} \mathrm{C} / 1.0 \mathrm{~kg} /\right.$ $\mathrm{cm}^{2}$ ). The sterilized media were then transferred into 42 $\mathrm{cm} \times 20 \mathrm{~cm} \times 16 \mathrm{~cm}(11.4 \mathrm{~L})$ plastic containers and allowed to cool prior to inoculation. Each container with the corn/ rye media were inoculated by cutting the PDA media containing $R$. solani into 1 to $2 \mathrm{~cm}$ squares. The PDA squares were transferred into the sterilized mixture and the tubs 
were covered with a lid and placed in a growth environment of $25-30{ }^{\circ} \mathrm{C}$ and $45 \%$ relative humidity. The fungi were grown in the sterilized mixture for 3-5 days until the presence of white-bodied sclerotia were noted. The media containing sheath blight pathogen were then air dried and ground. A total of $90 \mathrm{~kg}$ of inoculation media was produced and used for inoculation for each year.

\section{Plants and Data Collection}

Advanced-generation recombinant inbred line (RIL) lines derived from two weed $\times$ crop crosses were used in the study. The two weedy rice parents of the mapping populations were an indica-like straw hull accession (PI 653435; also known as AR-2001-1135-01, and RR9) and an aus-like black hull awned accession (PI 653419; also known as MS1996-9, and RR20). The two varieties were crossed with the indica landrace accession Dee Geo Woo Gen (DGWG) to produce two $\mathrm{F}_{2}$ mapping populations by (Thurber et al. 2013). These lines were then self-fertilized for 6 generation to create two $\mathrm{F}_{8}$ RIL populations totaling 184 lines from the $\mathrm{SH} \times \mathrm{DGWG}$ cross (referred to hereafter as the $\mathrm{S}$ population) and 236 lines from the BHA $\times$ DGWG cross (hereafter, the $\mathrm{B}$ population). Genotyping of the RILs occurred in the $F_{5}$ generation as described previously (Qi et al. 2015). In the 2015 and 2016 field season each RIL was planted with drill sowing in three rows that were $1.5 \mathrm{~m}$ long with a $0.6 \mathrm{~m}$ alley separating them. Each line was planted in three replicates in a complete block design where each block was one replication. The cultivar Lemont was planted as a border.

Following the protocol of Liu et al. 2013, approximately $50 \mathrm{~g}$ of $R$. solani (AG1-IA, teleomorph: Thanatephorus cucumeris) was spread per line along the bottom of the middle row in between the plant tillers at the water plant interface, 72 days after planting. Twenty-four days after inoculum was spread when the plants were in the heading and early flowering stages, each line was rated on a 1-9 scale. The entire middle row of plants was observed from the base of the plant to the panicle. For every $10 \%$ of the plant covered in lesions, the score was increased by an increment of 1 (e.g. $10 \%=1,20 \%=2, \ldots, 90 \%=9)$. A line was also scored as a 9 if the infection reached the panicle. $\mathrm{PH}$ and HD phenotypes were obtained from the 2012 field season. PH was measured at 100 days after emergence. HD measurements were obtained from (Qi et al. 2015).

\section{Genotyping}

Modified versions of the $F_{5}$ linkage maps for both populations generated by Qi et al. 2015 were used in this study. To remove problem markers we filtered SNPs if they had $>10 \%$ missing data or minor allele frequency $<$ $20 \%$. Additionally SNP positions were updated from the MSU 6.0 genome assembly to their MSU 7 positions using an in-house script. Final SNP counts are 4733 for the $\mathrm{S}$ population and 11,853 for the $\mathrm{B}$ population.

\section{QTL Analysis}

Analyses were performed in $\mathrm{R}$ with the $R / q t$ package (Broman et al. 2003) using a forward stepwise model fitting method with the Haley-Knott algorithm (Haley and Knott 1992). QTL were considered significant if their LOD score was higher than 3. Analyses for each year and mapping population were first performed without phenotypic covariates. Then each analysis was repeated using $\mathrm{PH}$ and HD as covariates. All possible interactions between QTL and covariates were tested. Any interactions above LOD 2 were included in the model because the risks associated with multiple hypothesis testing are lower with fewer comparisons. Moreover, identifying a false positive interaction between QTL that are known to be significant is less problematic than including a QTL that is actually a false positive. QTL positions were refined using the refineqtl function. The final LOD score and effect size of each QTL were calculated using a drop-one analysis within the fitqtl function. The 1-LOD support interval was calculated and visualized using the r/qtltools functions calcCis and segmentsOnMap respectively (Lovell 2017). Regions within the 1-Lod support interval of a QTL were searched for functionally characterized R genes in the QTARO database (Table S1) (Yamamoto et al. 2012).

\section{Phenotypic Analyses}

For both populations, the correlations between the 2015 and 2016 measures of ShB susceptibility were calculated using the $\mathrm{lm}$ function in $\mathrm{R}$. A model including the phenotypic covariates (and their potential interactions) in the absence of any genotypic markers was created for each population and year. The PVE and LOD for the full model and each individual phenotype and interaction were determined using the drop-one analysis within the fitqtl function.

\section{Results \\ Phenotypes}

Raw phenotypic data for the $\mathrm{B}$ and $\mathrm{S}$ populations are given in Table S2 and Table S3, respectively. The distribution of $\mathrm{ShB}$ resistance in each year and mapping population is presented in Fig. 1a-d. Sheath blight resistance was correlated between the 2015 and 2016 field season in both the $\mathrm{B}$ and $\mathrm{S}$ population $\left(R^{2}=0.25, p<\right.$ 0.001 ; and $R^{2}=0.47, p<0.001$ respectively). Both weed parents showed lower susceptibility than the crop parent. The cultivated parent DGWG had an average ShB susceptibility score of 5.3 in 2015 and 3.7 in 2016. The BHA parent had scores of 3.5 and 2.9 in 2015 and 2016, respectively. The SH parent scored 2.5 in 2015 and 2.3 in 2016. For the RILs, the average ShB susceptibility 

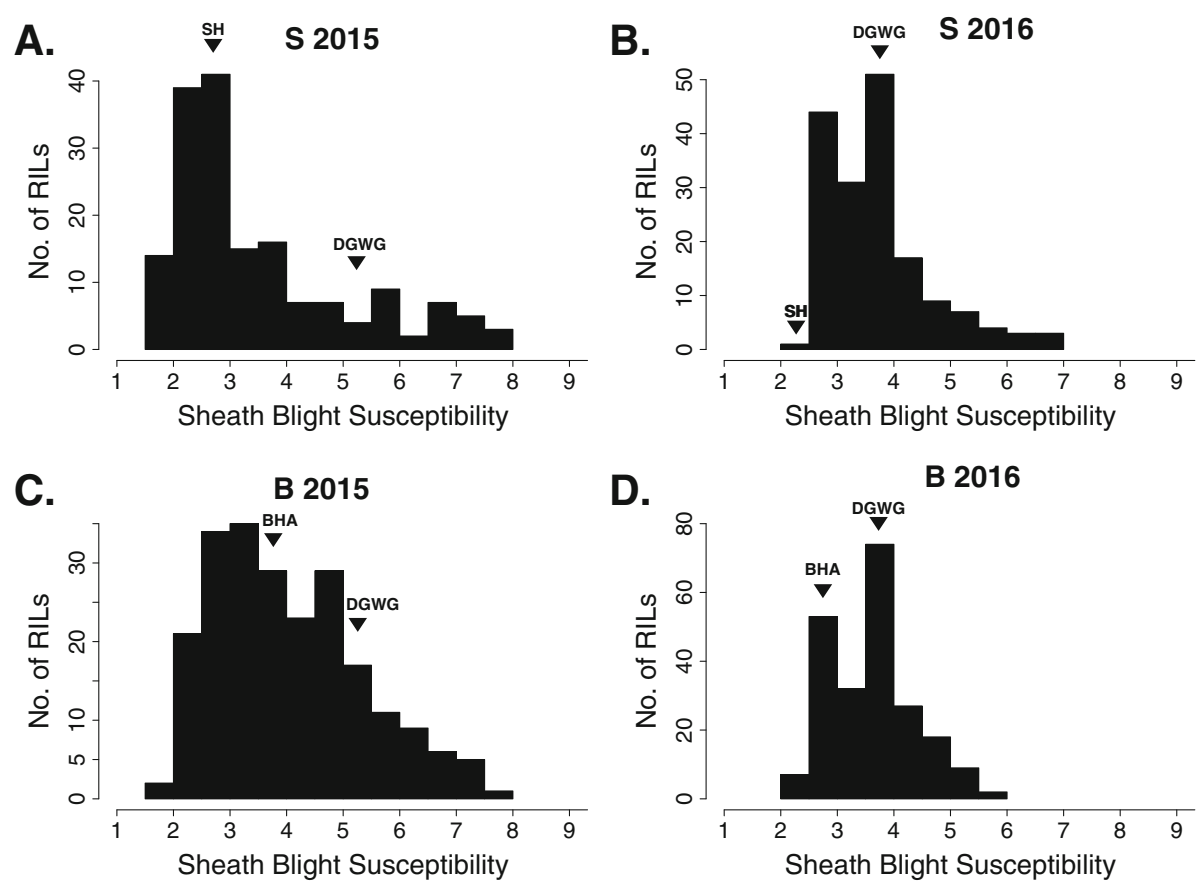

Fig. 1 Distribution of sheath blight susceptibility phenotypes in two F RIL populations. Histograms are shown for the S population in 2015 (a) and 2016 (b), and the B population in 2015 (c) and 2016 (d). Susceptibility was measured on a 1-9 scale from least to most infected. The phenotypes of the parents of each population are marked with arrows. DGWG = Dee-Geo-Woo-Gen, BHA = Black-hull awned, SH=Straw-hull

scores for the B population were 4.1 and 3.7 in 2015 and 2016, respectively. The average scores for the $\mathrm{S}$ population were 3.6 and 3.8 in 2015 and 2016, respectively. Transgressive segregation was more prevalent in 2015 for both populations. The B population had 80 RILs more resistant than BHA (36\% of all lines) and 36 more susceptible than DGWG (16\% of all lines). The S population had 36 RILs more resistant than SH (21\% of all lines) and 27 more susceptible than DGWG (16\% of all lines). The genotypes of the $10 \%$ most resistant RILs in 2015 for each population at each of our putative QTL are presented in Table S4; these genotypes may be of particular interest for breeding.

The combined effects of $\mathrm{PH}$ and HD explain $37.6 \%$ of the $\mathrm{ShB}$ variance in the B population in 2015 and $32.8 \%$ in 2016 (Table 1). There was no significant interaction between the two growth-related phenotypes in either year ( $p=0.16$; and $p=0.76$ in 2015 and 2016 respectively). In the $\mathrm{S}$ population, a model consisting of $\mathrm{PH}, \mathrm{HD}$ and their significant interaction explained $58.2 \%$ of the ShB resistance variance in 2015 and $51.3 \%$ in 2016 (Table 1).

\section{QTL Analysis}

\section{B Population}

Raw genotype data for the B population can be found in Table S5. Figure 2a shows the 1-LOD support intervals for both models in each year for the B population. When the QTL analysis was performed without phenotypic
Table 1 Results of models explaining variance in sheath blight resistance using only phenotypic covariates. The full model represents the additive effects of plant height, heading date and any significant interaction. The statistics for each individual component of the model are based on a drop-one analysis

\begin{tabular}{llll}
\hline Dataset & Model components & LOD $^{\mathrm{a}}$ & PVE $(\%)^{\mathrm{b}}$ \\
\hline B 2015 & Full model & 22.4 & 37.6 \\
& Plant height & 14.3 & 22.0 \\
& Heading date & 8.6 & 12.3 \\
B 2016 & Full model & 18.9 & 32.8 \\
& Plant height & 16.5 & 27.8 \\
& Heading date & 2.1 & 3.0 \\
S 2015 & Full model & 32.1 & 58.2 \\
& Plant height & 31.4 & 56.6 \\
& Heading date & 7.5 & 9.5 \\
& Plant height x Heading date & 5.4 & 6.6 \\
S 2016 & Full model & 26.6 & 51.3 \\
& Plant height & 22.5 & 40.8 \\
& Heading date & 14.7 & 23.7 \\
& Plant height x Heading date & 6.8 & 9.8 \\
\hline
\end{tabular}

aLogarithm of Odds

${ }^{\mathrm{b}}$ Percent of variance explained 


\section{A.}

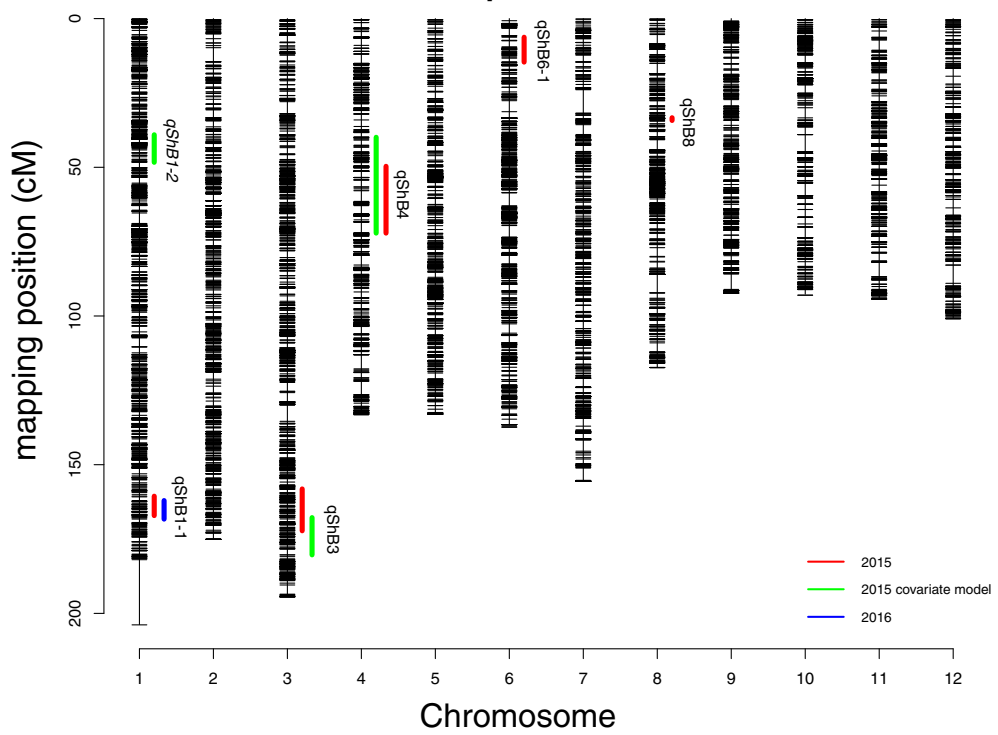

B.

S Population

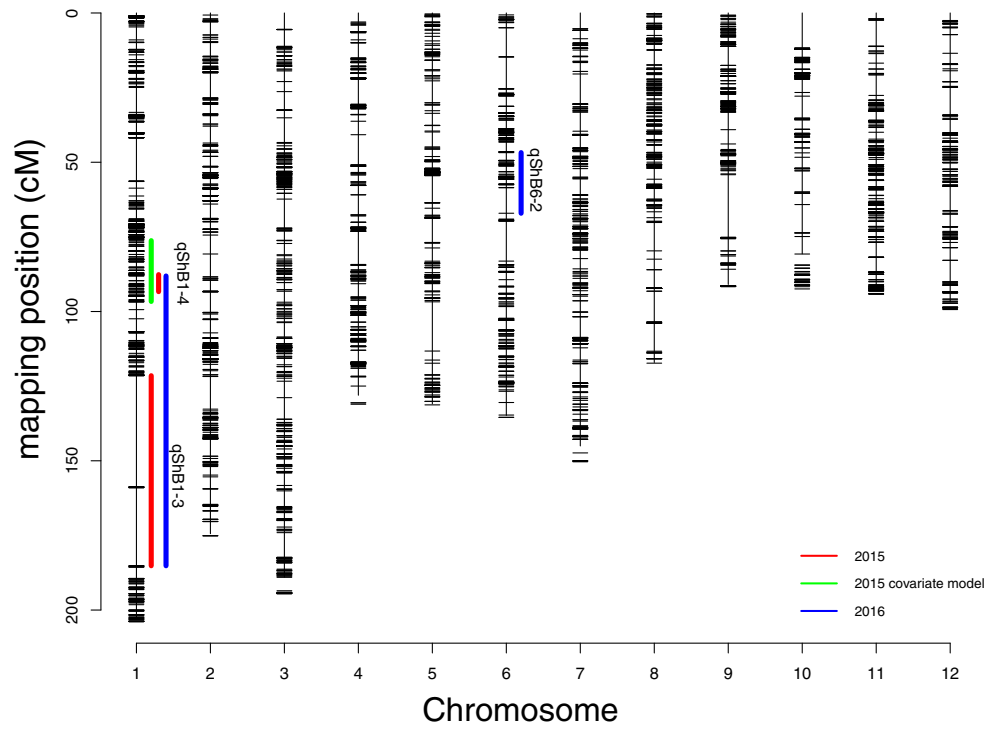

Fig. 2 High-resolution genetic map of the B population (a) and S population (b). Vertical lines represent each chromosome and horizontal dashes represent SNP markers. Genetic distances are measured in centimorgans. Bars represent the 1-LOD confidence interval of a QTL. Color indicates the year and mapping model. There were no significant QTL in 2016 for the model with covariates

covariates using the $2015 \mathrm{ShB}$ resistance data, five QTL were identified (indicated by red bars in Fig. 2a). Two of these, $q S h B 1-1$ and $q S h B 8$, had very large effects on the phenotype, with the weedy allele conferring an increased resistance at both loci (Table 2). However, both of these QTL co-localized with known $\mathrm{PH}$ and HD genes $s d 1$ and DTH8, respectively (Sasaki et al. 2002; Wei et al. 2010), and both disappeared when the analysis was performed using $\mathrm{PH}$ and $\mathrm{HD}$ as covariates. A small-effect QTL (qShB6-1) also co-localizes with a HD QTL previously identified in the B population (Qi et al. 2015) and was also removed. With the PH and HD QTL factored out, two small-effect ShB QTL remained significant ( $q S h B 3$ and $q S h B 4$ ) and one new QTL was detected on chromosome 1 ( $q S h B 1-2)$ (indicated in green; Fig. 2a). Notably, each of these three putative QTL contained at least one functionally characterized R-gene. The loci $q S h B 4$ and qShB1-2 contained OsSERK1 and OsRac1, respectively, while $q S h B 3$ contained both OsCPK1O and phyC. Each of these genes is annotated as being involved 
Table 2 Sheath blight resistance QTL identified in the two mapping populations in 2015 and 2016

\begin{tabular}{|c|c|c|c|c|c|c|c|c|c|}
\hline Model & QTL or covariate ${ }^{a}$ & Chromosome & $\operatorname{LOD}^{\mathrm{b}}$ & $\operatorname{PVE}(\%)^{c}$ & Effect & $\begin{array}{l}\text { Allele with increased } \\
\text { resistance }\end{array}$ & 1-LOD start ${ }^{d}$ & 1-LOD stop ${ }^{d}$ & Candidate gene \\
\hline \multicolumn{10}{|l|}{ B population } \\
\hline \multirow[t]{5}{*}{2015} & qShB1-1 & 1 & 14.26 & 18.26 & -0.61 & weed & $37,154,608$ & $38,207,165$ & sdl \\
\hline & qShB8 & 8 & 9.61 & 11.71 & -0.46 & weed & $4,097,722$ & $4,604,002$ & DTH8 \\
\hline & qShB3 & 3 & 4.60 & 5.32 & 0.32 & crop & $30,382,168$ & $33,046,007$ & \\
\hline & qShB4 & 4 & 4.50 & 5.19 & -0.31 & weed & $20,697,856$ & $23,609,792$ & \\
\hline & qShB6-1 & 6 & 3.59 & 4.10 & 0.28 & crop & $2,046,269$ & $3,171,640$ & $\mathrm{Hd} 3 \mathrm{a}$ \\
\hline \multirow{7}{*}{$\begin{array}{l}2015 \text { with } \\
\text { covariates }\end{array}$} & $\mathrm{PH}$ & & 17.94 & 21.55 & -0.03 & & & & \\
\hline & $\mathrm{HD}$ & & 11.70 & 13.11 & -0.06 & & & & \\
\hline & qShB3 & 3 & 7.02 & 7.48 & 0.38 & crop & $33,657,106$ & $35,750,342$ & \\
\hline & qShB1-2 & 1 & 5.71 & 6.00 & -0.21 & weed & $7,173,743$ & $38,433,096$ & \\
\hline & qShB4 & 4 & 3.71 & 3.82 & -0.18 & weed & $20,697,856$ & $23,609,792$ & \\
\hline & qShB1-2 x qShB4 & & 2.30 & 2.34 & 0.21 & & & & \\
\hline & qShB3-1 x HD & & 3.65 & 3.68 & -0.03 & & & & \\
\hline 2016 & qShB1-3 & 1 & 13.27 & 24.06 & -0.39 & weed & $37,310,074$ & $38,481,437$ & \\
\hline \multicolumn{10}{|l|}{ S population } \\
\hline \multirow[t]{2}{*}{2015} & qShB1-3 & 1 & 12.83 & 24.40 & -0.84 & weed & $28,586,738$ & $40,526,762$ & sdl \\
\hline & qShB1-4 & 1 & 3.12 & 5.16 & -0.38 & weed & $22,783,068$ & $23,642,407$ & \\
\hline \multirow{4}{*}{$\begin{array}{l}2015 \text { with } \\
\text { covariates }\end{array}$} & $\mathrm{PH}$ & & 32.29 & 53.03 & -0.24 & & & & \\
\hline & $\mathrm{HD}$ & & 7.69 & 8.76 & -0.24 & & & & \\
\hline & qShB1-4 & 1 & 3.86 & 4.17 & -0.33 & weed & $20,454,249$ & $24,016,179$ & \\
\hline & $\mathrm{PH} \times \mathrm{HD}$ & & 5.65 & 6.26 & 0.00 & & & & \\
\hline \multirow[t]{2}{*}{2016} & qShB1-3 & 1 & 5.99 & 13.76 & -0.34 & weed & $22,920,401$ & $40,526,762$ & $s d 1$ \\
\hline & qShB6-2 & 6 & 5.02 & 11.39 & 0.32 & crop & $8,057,485$ & $16,481,013$ & hdl \\
\hline
\end{tabular}

aPH, Plant height; HD, Heading date

bLOD, Logarithm of Odds

CPVE, Percent of the phenotypic variance explained

${ }^{\mathrm{d}}$ Genomic position based on MSU7 assembly

in blast disease resistance (Ono et al. 2001; Hu et al. 2005; Xie et al. 2011; Fu et al. 2013). A weak interaction between $q \operatorname{ShB1} 1-2$ and $q \operatorname{ShB} 4$ was found, as well as a moderate interaction between $q S h B 3$ and HD. The only QTL identified in the 2016 data was $q S h B 1-1$ (indicated in blue; Fig. 2a); it was significant when plant growth covariates were included, as it did for the 2015 data.

\section{$S$ Population}

Raw genotype data for the $\mathrm{S}$ population can be found in Table S6. Two QTL were identified in the S population on chromosome 1 in the model without covariates for the 2015 ShB resistance data (qShB1-3 and qShB1-4) (indicated in red; Fig. 2b). When $\mathrm{PH}, \mathrm{HD}$, and their interaction were included as covariates, $q \operatorname{ShB} 1-3$ was no longer significant while $q S h B 1-4$ remained significant (indicated in green; Fig. 2b). For the 2016 field data, one new QTL was discovered ( $q$ ShB6-2) (indicated in blue; Fig. 2b). This is a large-effect QTL that was only identified in the model without covariates and which contains hd1, a known HD gene (Yano et al. 2000). The single putative resistance QTL (qShB1-4) contained one functionally characterized fungal R-gene, OsACO7, which is annotated as a blast resistance gene (Iwai et al. 2006).

\section{Discussion}

Identifying QTL associated with increased resistance to ShB is of vital importance for increasing rice yields worldwide. One of the most challenging aspects of this search is to identify loci conferring resistance to the disease as contrast to those reflecting correlated growth traits $(\mathrm{PH}$ and HD). Our explicit incorporation of these confounding traits as covariates in the mapping model allowed us to accurately infer how each QTL contributes to ShB resistance and identify putative resistance QTL for further study. Additionally, the SNP linkage maps used in our analyses allowed us to identify functionally characterized candidate genes within each of the QTL. The close relationship between $\mathrm{PH}, \mathrm{HD}$, and $\mathrm{ShB}$ resistance that we confirm in this study also has implications for ShB management. 


\section{ShB QTL Explained by Plant Height or Heading Date}

The observed difference in ShB resistance between DGWG and weedy rice appears to primarily be explained by loci controlling $\mathrm{PH}$ and HD. This is not surprising, given previous work demonstrating differences in $\mathrm{PH}$ and HD between the parents of the mapping population (Reagon et al. 2011; Thurber et al. 2013; Qi et al. 2015). In both the B and S mapping populations, the single greatest contributor to sheath blight resistance was a QTL located on chromosome 1 ( $q S h B 1-1$ and $q S h B 1-3$ respectively). This QTL contains the famous green revolution gene semidwarf1 ( $s d 1)$, and the influence of this locus was removed in both populations when PH was used as a covariate. Our crop parent, DGWG, is the source of the original $s d 1$ allele that confers reduced plant height without loss of yields, whereas both weed parents have the wild-type, non-dwarf allele (Reagon et al. 2011; Thurber et al. 2013; $\mathrm{Li}$ et al. 2017). Therefore, it is reasonable to assume that this QTL is only indirectly related to ShB resistance due to its strong effect on $\mathrm{PH}$.

Both the B and S population possessed unique QTL for ShB resistance that appear to be due to effects on HD. All three of these QTL contained HD genes known to be involved in the HD difference between the crop and weed parents. Specifically, qShB6-2 contains hd1 which has a loss of function mutation in the weed, qShB8 contains DTH8 which has a loss of function mutation in the crop parent, and qShB6-1 contains $H d 3 a$ which has variation in the promoter that is associated with HD in both weeds and crops (Thurber et al. 2013, 2014; Qi et al. 2015). The PH QTL can also be assumed to be only indirectly related to ShB resistance, in this case, through their effect on HD.

\section{QTL Conferring Resistance to ShB}

Of the loci that remained significant after including $\mathrm{PH}$ and HD as covariates, qShB3 had the highest LOD score (8.1) and explained the largest percentage of the phenotypic variation (8.6\%) (Table 2). This QTL was only significant in the B mapping population, and the weed allele conferred reduced resistance. It is located near a previously identified ShB resistance QTL in other mapping populations, suggesting that the DGWG allele, which confers increased resistance, may be present in other germplasm (Zou et al. 2000; Liu et al. 2009). The locus $q$ ShB1-4 from the S population may also overlap with a previously identified QTL (Channamallikarjuna et al. 2010; Jia et al. 2012). Notably, it does not overlap with $q S h B 1-2$ from the B population. It is difficult to make exact position comparisons between linkage maps generated using SNP markers and earlier maps based on SSRs, so it is impossible to say for certain that these QTL represent those found in the prior studies. Even if they do overlap, however, it is still possible that the alleles responsible for increased resistance are unique to our study because of the divergence between weedy rice and the parents of the previous mapping population and germplasm used for genome wide association studies. The remaining two QTL (qShB1-2 and qShB4) have small effects but both provide resistance with the weedy allele and have not been previously reported in the literature.

Each of the four putative resistance QTL contained at least one functionally characterized fungal resistance gene; however, all of them were annotated as blast resistance genes. Similarly, the major sheath blight resistance QTL qShB9-2 in an indica rice variety Jasmine 85 was mapped at SSR marker RM245 (Liu et al. 2009). The blast resistance QTL qBLAST9.3 in Jasmine 85 was mapped between SSR markers RM107 and RM245 (Jia and Liu 2011) suggesting that genomic region at or nearby RM245 may harbor genes important for both rice blast and sheath blight disease resistance. It is possible that some of these genes play a more general role in fungal resistance, but further studies are required to determine whether these genes or previously uncharacterized loci occurring nearby are responsible for the observed effects on $\mathrm{ShB}$ resistance.

Notably, none of these loci were identified with the data from the 2016 field season. This result suggests that there are strong environmental effects acting on ShB, as has been previously reported (Zou et al. 2000; Channamallikarjuna et al. 2010). Our inability to detect ShB resistance QTL in 2016 could partially be due to lower overall levels of phenotypic variation in ShB resistance compared with 2015. This is possibly due to fewer of the RILs showing highly infected scores (e.g., >5) (see Fig. $1 \mathrm{~b}$ and d). The phenotypic distributions in 2016 were also bimodal, which was consistent with our observation that one QTL, controlled by $s d 1$, explained the largest proportion of the variance. This phenotypic difference between years suggests that the environmental conditions leading to genotypespecific ShB susceptibility in 2015 were not present in 2016. The 2016 field season experienced extensive rainfall, which resulted in the lodging of some plants during the disease evaluation period. It is possible that this introduced additional phenotypic variance which impaired our ability to map traits from that season.

\section{Utilizing Phenotypic Covariates to Map ShB Resistance QTL}

Previous studies of ShB resistance QTL have struggled to deal with the effects of $\mathrm{PH}$ and HD, and the common methods employed to deal this issue have had serious drawbacks (Zeng et al. 2015). By including these confounding traits as covariates in our mapping models we were able to overcome these issues. The primary method for dealing with $\mathrm{PH}$ and $\mathrm{HD}$ in the literature is to ignore any QTL that co-localizes with a PH or HD QTL (Zeng et al. 2015). This method is flawed because it requires that the correlated QTL have previously been identified 
and that a correlated QTL does not co-localize with a resistance QTL by chance. This is a serious problem considering that $\mathrm{PH}$ and HD QTL with large confidence intervals can be found across the whole rice genome. By measuring these traits alongside $\mathrm{ShB}$ resistance and including them as covariates, we can be more confident that the remaining QTL are actually involved in ShB resistance.

Another common method is to choose parents for the mapping population that are similar in $\mathrm{PH}$ and HD (Liu et al. 2013). This has two potential downsides. First, these traits could be polygenic and show transgressive segregation in the mapping population. This would result in a population that still shows strong correlations between ShB resistance and plant growth traits despite the parental similarity. The second downside is that it limits the potential for creating diverse mapping populations if only plants of similar growth characteristics can be chosen as parents. By including confounding plant growth traits as covariates in our mapping models, it is possible to map ShB resistance QTL using crosses between parents with drastically different morphological characteristics. Despite the morphological differences between the parents of our populations, we were still able to map multiple putative ShB resistance QTL. The fact that three of our four QTL increased resistance with the weedy allele indicates that these crop $x$ weed crosses can be valuable tools for breeding purposes and are an underutilized source of novel genes.

One potential caveat to note in our analyses is that the $\mathrm{PH}$ and HD data were collected in the 2012 field season, while ShB resistance was measured in 2015 and 2016. Despite the fact that the same lines were used in all three growing seasons, it is possible $\mathrm{PH}$ and $\mathrm{HD}$ may vary substantially with year and confound mapping. This is unlikely, however, as both traits are highly heritable (Han et al. 2017). Additionally, the large percentage of the variance in $\mathrm{ShB}$ resistance that they can explain and the fact that their use as covariates masks known $\mathrm{PH}$ and HD QTL implies that their inclusion in our model is appropriate. It is also possible that $R$. solani infection has an impact on $\mathrm{PH}$ (i.e. that highly susceptible plants are shorter due to negative effects of infection). Because we wanted to test how $\mathrm{PH}$ impacts $\mathrm{ShB}$ resistance, not how ShB resistance impacts $\mathrm{PH}$, it was important to collect $\mathrm{PH}$ data in the absence of fungal infection.

\section{Implications for Breeding and Variety Selection}

Our observation that the difference in ShB resistance between cultivated and weedy rice is mostly due to $\mathrm{PH}$ and HD has some implications for the disease management in the region. It is possible that by choosing taller crop varieties with later heading dates, rice farmers may be able to increase their yield in fields chronically affected by sheath blight and weedy rice. Despite the large number of QTL identified in previous studies, few if any have been utilized in breeding programs because they tend to have small effects (Zeng et al. 2015). It has been suggested that breeding programs should stop waiting for large-effect loci to be discovered and instead begin breeding lines that pyramid multiple small-effect loci in a single genetic background. Utilizing an approach that emphasizes growing varieties with favorable plant architectures and multiple small to moderate-effect QTL, such as those identified in this study, is likely the best way to combat $\mathrm{ShB}$. The blast resistance genes occurring within the ShB resistance QTL identified here and previously may be good candidates for future studies (Liu et al. 2009; Jia and Liu 2011). Next steps in this system include fine mapping with near isogenic lines and functional verification of candidate genes.

\section{Conclusions}

We identified four putative ShB resistance QTL that were not associated with $\mathrm{PH}$ or HD, two of which have not been reported in the literature. These QTL can be used in combination with other small to moderate effect resistance QTL to breed for more disease resistant rice varieties. Additionally, our approach of using $\mathrm{PH}$ and $\mathrm{HD}$ as covariates in our mapping models can be a powerful tool for identifying ShB resistance QTLs in future studies.

\section{Supplementary information}

Supplementary information accompanies this paper at https://doi.org/10. 1186/s12284-020-00381-9.

Additional file 1: Table S1. Functionally characterized genes from the QTARO database. Candidate genes for each QTL are highlighted in yellow.

Additional file 2: Table S2. Phenotyping data for the B population including ShB susceptibility in 2015 and 2016, PH in 2012, and HD in 2012. (CSV 4 kb)

Additional file 3: Table S3. Phenotyping data for the $\mathrm{S}$ population including ShB susceptibility in 2015 and 2016, PH in 2012, and HD in 2012. (CSV 3 kb)

Additional file 4: Table S4. The genotypes of the top 10\% most resistant RILs in the 2015 season at each of our putative QTL.

Additional file 5: Table S5. Genotyping data of RILs in the B population generated using genotyping-by-sequencing in the $F_{5}$ generation. (CSV $5483 \mathrm{~kb}$ )

Additional file 6: Table S6. Genotyping data of RILs in the S population generated using genotyping-by-sequencing in the $F_{5}$ generation. (CSV $1736 \mathrm{~kb})$

\section{Abbreviations}

BHA: black hull awned; DGWG: Dee-Geo-Woo-Gen; HD: heading date; LOD: logarithm of odds; PDA: potato dextrose agar; PH: plant height; QTL: quantitative trait locus; RIL: recombinant inbred line; SH: straw hull; ShB: Sheath blight; SNP: single nucleotide polymorphism

\section{Acknowledgements}

We thank Tracy Bianco, Heather Herald, Howard Black, LaDuska Sells,

Jianbing Ma, Michael Lin, Tiffany Sookaserm, Bill Luebke, Tyler Franzen, Jenny 
C. Jia, and other staff members of DB NRRC for their excellent technical support. The United States Department of Agriculture is an equal opportunity provider and employer.

\section{Authors' Contributions}

DMG performed all of the analyses, interpreted the results, drafted the manuscript and designed the Figs. YJ planned and designed the experiment. $Y J, A G$ and $Y L$ performed field experiments. YJ, KMO, AC and DG supervised the project and contributed to the manuscript. The author(s) read and approved the final manuscript.

\section{Authors' Information}

Not applicable.

\section{Funding}

This project was funded, in part, by the National Science Foundation Plant Genome Research Program (NSF award IOS-1032023).

\section{Availability of Data and Materials}

All data generated or analyzed during this study are included in this published article and its supplementary information files.

\section{Ethics Approval and Consent to Participate}

Not applicable.

\section{Consent for Publication}

Not applicable.

\section{Competing Interests}

The authors declare that they have no competing interests.

\section{Author details}

'Department of Biology, Washington University in St. Louis, 1 Brookings Drive, Campus Box 1137, St. Louis, MO 63110, USA. ${ }^{2}$ United States Department of Agriculture-Agricultural Research Service, Dale Bumpers National Rice Research Center, 2890 HWY 130 E, Stuttgart, AR 72160, USA ${ }^{3}$ University of Arkansas Rice Research and Extension Center, 2900 AR-130, Stuttgart, AR 72160, USA. ${ }^{4}$ Present address: Arkansas Department of Health, Little Rock, AR 72205, USA. ${ }^{5}$ Present address: Department of Plant Pathology, Washington State University, Pullman, WA 99164, USA. ${ }^{6}$ Department of Biology, University of Massachusetts, Amherst, USA.

\section{Received: 30 September 2019 Accepted: 6 March 2020}

Published online: 23 March 2020

\section{References}

Broman KW, Wu H, Sen S, Churchill GA (2003) R/QTL: QTL mapping in experimental crosses. Bioinformatics 19:889-890. https://doi.org/10.1093/ bioinformatics/btg112

Channamallikarjuna V, Sonah $\mathrm{H}$, Prasad M et al (2010) Identification of major quantitative trait loci qSBR11-1 for sheath blight resistance in rice. Mol Breed 25:155-166. https://doi.org/10.1007/s11032-009-9316-5

Eizenga GC, Jia MH, Pinson SR et al (2015) Exploring sheath blight quantitative trait loci in a Lemont/O. meridionalis advanced backcross population. Mol Breed 35:140. https://doi.org/10.1007/s11032-015-0332-3

Eizenga GC, Prasad B, Jackson AK, Jia MH (2013) Identification of rice sheath blight and blast quantitative trait loci in two different 0 . sativa/O. nivara advanced backcross populations. Mol Breed 31:889-907

Fu L, Yu X, An C (2013) Overexpression of constitutively active OsCPK10 increases Arabidopsis resistance against Pseudomonas syringae $\mathrm{pv}$. Tomato and rice resistance against Magnaporthe grisea. Plant Physiol Biochem 73:202-210. https://doi.org/10.1016/j.plaphy.2013.10.004

Haley CS, Knott SA (1992) A simple regression method for mapping quantitative trait loci in line crosses using flanking markers. Heredity 69:315-324. https:// doi.org/10.1038/hdy.1992.131

Han Z, Hu W, Tan C, Xing Y (2017) QTLs for heading date and plant height under multiple environments in rice. Genetica 145:67-77. https://doi.org/10.1007/ s10709-016-9946-6

Hu H, Xiong L, Yang Y (2005) Rice SERK1 gene positively regulates somatic embryogenesis of cultured cell and host defense response against fungal infection. Planta 222:107-117. https://doi.org/10.1007/s00425-005-1534-4
Iwai T, Miyasaka A, Seo S, Ohashi Y (2006) Contribution of ethylene biosynthesis for resistance to blast fungus infection in young Rice plants. Plant Physiol 142:1202-1215. https://doi.org/10.1104/pp.106.085258

Jia L, Yan W, Zhu C et al (2012) Allelic analysis of sheath blight resistance with association mapping in Rice. PLoS One 7:e32703. https://doi.org/10.1371/ journal.pone.0032703

Jia Y, Liu G (2011) Mapping quantitative trait loci for resistance to rice blast. Phytopathol. 101:176-187. https://doi.org/10.1094/PHYTO-06-10-0151

Lee FN, Rush MC (1983) Rice sheath blight: a major rice disease. Plant Dis 67:829-832

Li L-F, Li Y-L, Jia Y et al (2017) Signatures of adaptation in the weedy rice genome. Nat Genet 49:811-814. https://doi.org/10.1038/ng.3825

Li Z, Pinson SRM, Marchetti MA et al (1995) Characterization of quantitative trait loci (QTLs) in cultivated rice contributing to field resistance to sheath blight (Rhizoctonia solani). Theor Appl Genet 91:382-388. https://doi.org/10.1007/ BF00220903

Liu G, Jia Y, Correa-Victoria FJ et al (2009) Mapping quantitative trait loci responsible for resistance to sheath blight in Rice. Phytopathology 99:10781084. https://doi.org/10.1094/PHYTO-99-9-1078

Liu G, Jia Y, McClung A et al (2013) Confirming QTLs and finding additional loci responsible for resistance to Rice sheath blight disease. Plant Dis 97:113-117. https://doi.org/10.1094/PDIS-05-12-0466-RE

Liu Y, Qi X, Gealy DR et al (2015) QTL analysis for resistance to blast disease in U. S. weedy Rice. Mol Plant-Microbe Interact 28:834-844. https://doi.org/10. 1094/MPMI-12-14-0386-R

Londo JP, Schaal BA (2007) Origins and population genetics of weedy red rice in the USA. Mol Ecol 16:4523-4535. https://doi.org/10.1111/j.1365-294X.2007.03489.x

Lovell J (2017) qtITools. https://github.com/jtlovell/qtITools

Molla KA, Karmakar S, Molla J et al (2019) Understanding sheath blight resistance in rice: the road behind and the road ahead. Plant Biotechnol J Accepted Author Manuscript. https://doi.org/10.1111/pbi.13312

Nelson JC, Oard JH, Groth D et al (2012) Sheath-blight resistance QTLS in japonica rice germplasm. Euphytica 184:23-34. https://doi.org/10.1007/ s10681-011-0475-1

Ono E, Wong HL, Kawasaki T et al (2001) Essential role of the small GTPase Rac in disease resistance of rice. Proc Natl Acad Sci 98:759-764. https://doi.org/10. 1073/pnas.98.2.759

Qi X, Liu Y, Vigueira CC et al (2015) More than one way to evolve a weed: parallel evolution of US weedy rice through independent genetic mechanisms. Mol Ecol 24:3329-3344. https://doi.org/10.1111/mec.13256

Reagon M, Thurber CS, Gross BL et al (2010) Genomic patterns of nucleotide diversity in divergent populations of U.S. weedy rice. BMC Evol Biol 10:180. https://doi.org/10.1186/1471-2148-10-180

Reagon M, Thurber CS, Olsen KM, et al (2011) The long and the short of it: SD1 polymorphism and the evolution of growth trait divergence in US weedy rice. Mol Ecol 2011 Sep;20(18):3743-3756. doi: https://doi.org/10.1111/j.1365294X.2011.05216.x

Sasaki A, Ashikari M, Ueguchi-Tanaka M et al (2002) Green revolution: a mutant gibberellin-synthesis gene in rice. Nature 416:701-702. https://doi.org/10. 1038/416701a

Savary S, Teng PS, Willocquet L, Nutter FW (2006) Quantification and modeling of crop losses: a review of purposes. Annu Rev Phytopathol 44:89-112. https:// doi.org/10.1146/annurev.phyto.44.070505.143342

Taguchi-Shiobara F, Ozaki H, Sato H et al (2013) Mapping and validation of QTLS for rice sheath blight resistance. Breed Sci 63:301-308. https://doi.org/10. $1270 / j s b b s .63 .301$

Thurber CS, Jia MH, Jia Y, Caicedo AL (2013) Similar traits, different genes? Examining convergent evolution in related weedy rice populations. $\mathrm{Mol}$ Ecol 22:685-698. https://doi.org/10.1111/mec.12147

Thurber CS, Reagon M, Olsen KM et al. (2014) The evolution of flowering strategies in US weedy rice. Amer J Bot 101:1737-1747

Wasano K, Hirota Y (1986) Varietal resistance of rice to sheath blight disease caused by Rhizoctonia solani Kuhn, by the syringe inoculation method. Bull Fac Agr Saga Univ 60:49-59

Wedger MJ, Olsen KM (2018) Evolving insights on weedy rice. Ecol Genet Genomics 7-8:23-26. https://doi.org/10.1016/j.egg.2018.03.005

Wei X, Xu J, Guo H et al (2010) DTH8 suppresses flowering in rice, influencing plant height and yield potential simultaneously. Plant Physiol pp 110(156943). https://doi.org/10.1104/pp.110.156943

Wrather JA Sweets L (2009) Rice sheath blight control. University of Missouri Extension. https://extension2.missouri.edu/mp646 
Xie X-Z, Xue Y-J, Zhou J-J et al (2011) Phytochromes regulate SA and JA signaling pathways in Rice and are required for developmentally controlled resistance to Magnaporthe grisea. Mol Plant 4:688-696. https://doi.org/10. $1093 / \mathrm{mp} / \mathrm{ssr} 005$

Xu Q, Yuan X, Yu H et al (2011) Mapping quantitative trait loci for sheath blight resistance in rice using double haploid population. Plant Breed 130:404-406. https://doi.org/10.1111/j.1439-0523.2010.01806.x

Yamamoto E, Yonemaru J-I, Yamamoto T, Yano M (2012) OGRO: the overview of functionally characterized genes in Rice online database. Rice N Y N 5:26. https://doi.org/10.1186/1939-8433-5-26

Yano M, Katayose Y, Ashikari M et al (2000) Hd1, a major photoperiod sensitivity quantitative trait locus in Rice, is closely related to the Arabidopsis flowering time gene CONSTANS. Plant Cell 12:2473-2483. https://doi.org/10.1105/tpc. 12.12.2473

Zeng Y, Ji Z, Yang C (2015) The way to a more precise sheath blight resistance QTL in rice. Euphytica 203:33-45. https://doi.org/10.1007/s10681-014-1246-6

Zou JH, Pan XB, Chen ZX et al (2000) Mapping quantitative trait loci controlling sheath blight resistance in two rice cultivars (Oryza sativa L.). Theor Appl Genet 101:569-573. https://doi.org/10.1007/s001220051517

Zuo S, Yin Y, Pan C et al (2013) Fine mapping of qSB-11LE, the QTL that confers partial resistance to rice sheath blight. Theor Appl Genet 126:1257-1272. https://doi.org/10.1007/s00122-013-2051-7

Zuo S, Zhang Y, Yin Y et al (2014) Fine-mapping of qSB-9TQ, a gene conferring major quantitative resistance to rice sheath blight. Mol Breed 34:2191-2203

\section{Publisher's Note}

Springer Nature remains neutral with regard to jurisdictional claims in published maps and institutional affiliations.

\section{Submit your manuscript to a SpringerOpen ${ }^{\circ}$ journal and benefit from:}

- Convenient online submission

- Rigorous peer review

- Open access: articles freely available online

High visibility within the field

- Retaining the copyright to your article

Submit your next manuscript at $\boldsymbol{\nabla}$ springeropen.com 